\title{
INFINITELY MANY COEXISTING SINKS FROM DEGENERATE HOMOCLINIC TANGENCIES
}

\author{
GREGORY J. DAVIS
}

Dedicated to Clark Robinson

\begin{abstract}
The evolution of a horseshoe is an interesting and important phenomenon in Dynamical Systems as it represents a change from a nonchaotic state to a state of chaos. As we are interested in determining how this transition takes place, we are studying certain families of diffeomorphisms. We restrict our attention to certain one-parameter families $\left\{F_{t}\right\}$ of diffeomorphisms in two dimensions. It is assumed that each family has a curve of dissipative periodic saddle points, $P_{t} ; F_{t}^{n}\left(P_{t}\right)=P_{t}$, and $\left|\operatorname{det} D F_{t}^{n}\left(P_{t}\right)\right|<1$. We also require the stable and unstable manifolds of $P_{t}$ to form homoclinic tangencies as the parameter $t$ varies through $t_{0}$. Our emphasis is the exploration of the behavior of families of diffeomorphisms for parameter values $t$ near $t_{0}$. We show that there are parameter values $t$ near $t_{0}$ at which $F_{t}$ has infinitely many co-existing periodic sinks.
\end{abstract}

\section{INTRODUCTION AND STATEMENT OF MAIN RESULTS}

The transition from Morse-Smale systems to more complicated systems, i.e., those with a countable infinite set of periodic attractors, was studied by Gavrilov and Silnikov [3]. They discovered that if a $C^{3}$ family of diffeomorphisms creates nondegenerate tangencies, then there exists an infinite cascade of sinks associated with the tangency.

In addition to proving the same result independently, Newhouse [9] gave a negative answer to a conjecture of Smale [15]. Smale had conjectured that most diffeomorphisms on $S^{2}$ with the $C^{r}$ topology should have only finitely many sinks; Newhouse constructed a special set of diffeomorphisms to prove the following theorem:

Theorem. On any manifold $M$ of dimension greater than one, there is a residual subset $B$ of an open set in $\operatorname{Diff}^{r}(M), r \geq 2$, such that every element of $B$ has infinitely many sinks.

Later, Newhouse [10] proved that infinitely many sinks coexist for an open set of diffeomorphisms $B$ which surround a set of diffeomorphisms $F$ in the $C^{2}$ topology. He made the assumption that the diffeomorphisms $F$ could be

Received by the editors October 10, 1987 and, in revised form February 2, 1989.

1980 Mathematics Subject Classification (1985 Revision). Primary 58F13. 
perturbed to contain a dissipative periodic saddle point that has a nondegenerate tangency of stable and unstable manifolds. Robinson [13] supplied a key proposition that enabled him to show that Newhouse's result is true for fixed one parameter families of diffeomorphisms. The result is as follows:

Theorem. Suppose $\left\{F_{t}\right\}$ is a fixed one parameter family of $C^{3}$ diffeomorphisms of a two manifold which nondegenerately creates homoclinic intersections at $t_{0}$ for the periodic points $P_{t}$ of period $n$ with

$$
\left.\left|\operatorname{det} D F_{t_{0}}^{n}\left(P_{t_{0}}\right)\right|<1 \quad \text { (respectively }\left|\operatorname{det} D F_{t_{0}}^{n}\left(P_{t_{0}}\right)>1\right|\right) \text {. }
$$

Then, given $\varepsilon>0$, there exists a subinterval $\left[t_{1}, t_{2}\right] \subset\left[t_{0}-\varepsilon, t_{0}+\varepsilon\right]$ and a residual subset $J$ in $\left[t_{1}, t_{2}\right]$ such that for $t$ in $J, F_{t}$ has infinitely many sinks ( respectively sources).

In the same paper, Robinson also proved a theorem on the cascade of sinks for real analytic diffeomorphisms. His result is that a fixed one-parameter family of real analytic diffeomorphisms which create degenerate homoclinic tangencies have an infinite cascade of periodic sinks. He then applied this result to the Hénon map. Based on computer evidence that homoclinic tangencies occur when $B$ is -0.3 and $A$ is near 1.39 , Robinson conjectured:

Conjecture. The Hénon map, $F_{A B}(x, y)=\left(A-B y-x^{2}, x\right)$, has an infinite cascade of sinks for $b=-0.3$ and as $A$ varies from 1.39 to 1.4 .

Through numerical studies, Hénon [6] claims that the map $F_{A B}$ has a strange attractor when $A=1.4$ and $B=-0.3$. Newhouse [10] made the following conjecture about the Hénon map:

Conjecture. The Hénon map, $F_{A B}(x, y)=\left(A-B y-x^{2}, x\right)$, of the plane, does not have a transitive strange attractor, but instead, a long periodic orbit when $A=1.4$ and $B=-0.3$.

In order to prove this conjecture using the theorems of Newhouse [10] and Robinson [13], it is necessary not only to show that homoclinic tangencies occur, but also that they are in fact nondegenerate tangencies. Our result, however, eliminates the need to show that the tangencies are formed nondegenerately:

Main Result. Let $\left\{F_{t}\right\}$ be a fixed one parameter family of $C^{\infty}$ diffeomorphisms in two dimensions. Assume that at $t_{0},\left\{F_{t}\right\}$ creates finite order homoclinic tangencies for the periodic points $P_{t}$ of period $n$ with

$$
\left|\operatorname{det} D F_{t_{0}}^{n}\left(P_{t_{0}}\right)\right|<1 \quad\left(\left|\operatorname{det} D F_{t_{0}}^{n}\left(P_{t_{0}}\right)>1\right|\right),
$$

and that $F_{t}^{n}$ is $C^{\infty}$ linearizable in a neighborhood of $P_{t}$. Then, for $\varepsilon>0$, there exists a subinterval $\left[t_{1}, t_{2}\right] \subset\left[t_{0}-\varepsilon, t_{0}+\varepsilon\right]$ and a residual subset $J$ in $\left[t_{1}, t_{2}\right]$ such that for $t$ in $J, F_{t}$ has infinitely many sinks (sources).

If it can be shown that homoclinic tangencies do occur for the parameter values in question $(A \approx 1.39$ and $B=-0.3)$, then by combining our Main Result 
and the Conjecture below, positive answers to the conjectures of Newhouse [10] and Robinson [13] would be obtained.

Conjecture. For the Hénon map, homoclinic tangencies occur for parameter values $B=-0.3$ and $A \approx 1.39$.

While the possibility of infinitely many coexisting sinks occurring for the parameter values $A \approx 1.39$ and $B=-0.3$ remains open, the work of van Strien [16] has shown that for certain Hénon maps near a one-dimensional map, the phenomena of infinitely many coexisting sinks does indeed occur.

Definitions. Throughout this paper, we will let $\left\{F_{t}\right\}$ denote a fixed one parameter family of $C^{\infty}$ planar diffeomorphisms which depend on the real parameter $t$, (i.e., $F_{t}(\cdot): R^{2} \rightarrow R^{2}, t \in R$ ) and which have the following properties:

(a) $\left\{F_{t}\right\}$ has a curve of dissipative saddle points, $P_{t}$.

(b) New homoclinic tangencies of finite order for $P_{t}$ are created as the parameter $t$ varies through $t_{0}$.

(c) $F_{t_{0}}^{n}$ is $C^{\infty}$ linearizable in a neighborhood of $P_{t_{0}}$.

Before we can fully explain these conditions, it will be necessary to provide some basic definitions and facts.

For a diffeomorphism $F$, let $D F(p)$ denote the Jacobian derivative at $p$; that is, let $D F(p)$ be the matrix of partial derivatives. The orbit of $p$ is the set $O(p)=\left\{F^{n}(p): n \in Z\right\}$. When $O(p)$ is a finite set, $p$ is a periodic point of period $n$ if $n$ is the least positive integer such that $F^{n}(p)=p$. If $F(p)=p$, then $p$ is called a fixed point of $F$. A point $p$ is called a periodic sink if $p$ is a periodic point and all eigenvalues of $D F^{n}(p)$ have absolute value less than one. A point $p$ is called a periodic source if $p$ is a periodic point and all eigenvalues of $D F^{n}(p)$ have absolute value greater than one.

Let $p$ be a periodic point of period $n$ for a diffeomorphism $F$ in two dimensions, and let $\lambda$ and $\mu$ be the eigenvalues of $D F^{n}(p)$. If $\lambda$ and $\mu$ are both real and $|\lambda|>1$ and $|\mu|<1$, the point $p$ is called a periodic saddle point.

There are two smooth one-dimensional manifolds corresponding to a saddle point $p$ of $F$ :

(a) the stable manifold of $p$, which is defined by

$$
W^{s}(p, F)=\left\{q: d\left(F^{j}(q), F^{j}(p)\right) \rightarrow 0 \text { as } j \rightarrow \infty\right\},
$$

(b) the unstable manifold of $p$, which is defined by

$$
W^{u}(p, F)=\left\{q: d\left(F^{j}(q), F^{j}(p)\right) \rightarrow 0 \text { as } j \rightarrow-\infty\right\} .
$$

If $q \neq p$ and $q \in W^{s}(p, F) \cap W^{u}(p, F)$, then $q$ is called a homoclinic point. A homoclinic point can either be a point of transverse intersection between $W^{s}(p, F)$ and $W^{u}(p, F)$, or a point of tangency of $W^{s}(p, F)$ and $W^{u}(p, F)$. Precise definitions of these two concepts need to be made. Let $\gamma^{1}$ and $\gamma^{2}$ be two oriented differentiable curves which intersect at the point $q$. If there exist local 
coordinates $(x, y)$ near $q$ such that $x(q)=y(q)=0$ and in which there exist oriented parametrizations of $\gamma^{j}$ for $j=1,2$ of the form $\left\{\left(x_{j}(s), y_{j}(s)\right):|s|<\right.$ $\varepsilon\}$ which satisfy the following conditions:

(a) $y_{2}(s) \equiv 0, x_{2}(0)=0$, and $x_{2}^{\prime}(0)>0$,

(b) $y_{1}(0)=0=x_{1}(0), y_{1}(s)<0$ for $-\varepsilon<s<0$, and $y_{1}(s)>0$ for $0<s<\varepsilon$,

then curve $\gamma^{1}$ is said to have a positive intersection with curve $\gamma^{2}$ at point $q$. Similarly, a curve $\gamma^{1}$ is said to have a negative intersection with curve $\gamma^{2}$ at point $q$ if in the above definition condition (b) is replaced with

$$
\begin{aligned}
& \left(\mathrm{b}^{\prime}\right) \quad y_{1}(0)=0=x_{1}(0), y_{1}(s)<0 \text { for } 0<s<\varepsilon \text {, and } y_{1}(s)>0 \text { for } \\
& -\varepsilon<s<0 .
\end{aligned}
$$

The curves $\gamma^{1}$ and $\gamma^{2}$ have tangency of order $n$ if there are parametrizations as above such that (a) $y_{2}(s) \equiv 0$; and (b) $y_{1}(0), y_{1}^{\prime}(0), \ldots, y_{1}^{(n-1)}(0)=0$, but $y_{1}^{(n)}(0) \neq 0$.

At this point we can use the above ideas to provide a precise definition for the creation of homoclinic tangencies:

$\left\{F_{t}\right\}$ creates homoclinic tangencies at $t_{0} \quad\left(\left\{F_{t}\right\}\right.$ creates homoclinic intersections) for a periodic saddle point $P_{t}$ if $\varepsilon>0, Q_{t}=F_{t}^{k}\left(P_{t}\right)$ for some $k$, and there exist continuously varying subarcs $\gamma_{t}^{s} \subset W^{s}\left(P_{t}, F_{t}\right)$ and $\gamma_{t}^{u} \subset W^{u}\left(Q_{t}, F_{t}\right)$ for $t_{0}-\varepsilon \leq t \leq t_{0}+\varepsilon$, such that

(a) $\gamma_{t}^{s} \cup \gamma_{t}^{u}=\varnothing$ for $t_{0}-\varepsilon \leq t<t_{0}$, and

(b) for $t_{0}<t \leq t_{0}+\varepsilon, \gamma_{t}^{s}$ has both positive and negative intersections with $\gamma_{t}^{u}$.

$\left\{F_{t}\right\}$ creates homoclinic tangencies of finite order at $t_{0}$ if condition (a) above is satisfied, and condition (b) is replaced with

$\left(\mathrm{b}^{\prime}\right)$ for $t_{0}<t \leq t_{0}+\varepsilon, \gamma_{t}^{u}$ has at least one positive intersection with $\gamma_{t}^{s}$ of odd order and at least one negative intersection with $\gamma_{t}^{s}$ of odd order.

$\left\{F_{t}\right\}$ creates degenerate homoclinic tangencies of constant finite order at $t_{0}$, if $\left\{F_{t}\right\}$ creates homoclinic tangencies of finite order [conditions $(a)$ and $\left(\mathbf{b}^{\prime}\right)$ above] and

(c) $\gamma_{t_{0}}^{s}$ and $\gamma_{t_{0}}^{u}$ have tangency of even order $j$.

(d) If coordinates are taken so $\gamma_{t}^{s}$ lies on $y=0$ and $y^{*}(t)$ is the extreme value of $y$ along $\gamma_{t}^{u}$, then $d y^{*} / d t \neq 0$ at $t=0$.

(e) There is an interval of parameter values containing $t_{0}$ for which the lowest order terms remain $j$ th order at $y^{*}(t)$.

$\left\{F_{t}\right\}$ creates a nondegenerate tangency at $t_{0} \quad\left(\left\{F_{t}\right\}\right.$ nondegenerately creates homoclinic intersections at $\left.t_{0}\right)$ if $\left\{F_{t}\right\}$ creates homoclinic tangencies of finite order [conditions (a) and $\left(\mathbf{b}^{\prime}\right)$ above], and

(c') $\gamma_{t_{0}}^{s}$ and $\gamma_{t_{0}}^{u}$ have tangency of order two. 
(d) If coordinates are taken so $\gamma_{t}^{s}$ lies on $y=0$ and $y^{*}(t)$ is the extreme value of $y$ along $\gamma_{t}^{u}$, then $d y^{*} / d t \neq 0$ at $t=0$.

Hyperbolic invariant sets within a dynamical system are of great importance in the study of chaotic dynamics as these are sets which contain asymptotic behavior of a dynamical system. A horseshoe is an example of a hyperbolic invariant set and since horseshoes are formed whenever homoclinic intersections are formed (the Smale-Birkhoff Homoclinic Theorem [5, p. 252]), they are integral to our study. The Smale horseshoe is a prototypical model of a hyperbolic invariant set.

Recall that a set $S$ is invariant for $F$ if $F(S)=S$. Now assume there are integers $n$, boxes $B_{n}$, and parameter values $t=t_{n}$, such that $F_{t}^{n}\left(B_{n}\right)$ crosses $B_{n}$ in the shape of a horseshoe. Let $G=F_{t}^{n}$ and $B=B_{n}$, and define the set $\Lambda=\left\{G^{k}(B): k \in Z\right\}$ to be the maximal invariant set for $G$ in $B$; i.e., $\Lambda$ is the set of all points $q$ such that both the forward and backward orbit of $q$ by $G$ stays in $B$.

A closed invariant set $\Lambda$, defined by $G: R^{n} \rightarrow R^{n}$, is said to have a hyperbolic structure if there is a continuous direct sum decomposition, $T_{\Lambda} R=E_{\Lambda}^{s} \oplus E_{\Lambda}^{u}$, with the property that there are two constants $C>0$ and $\lambda>1$ such that for $k \geq 0$ and for $\nu^{s}$ in $E_{q}^{s},\left|D G^{k}(q) \nu^{s}\right| \leq \mathrm{C} \lambda^{-k}\left|\nu^{s}\right|$, and for $\nu^{u}$ in $E_{q}^{u}$, $\left|D G^{-k}(q) \nu^{u}\right| \leq \mathrm{C} \lambda^{-k}\left|\nu^{u}\right|$. According to the Stable Manifold Theorem for Hyperbolic Sets [7], the points of a hyperbolic invariant set are similar to hyperbolic saddle points.

$W_{\varepsilon}^{s}(x, G)$ is the local stable manifold of $x$ of size $\varepsilon . W_{\varepsilon}^{u}(x, G)$ is the local unstable manifold of $x$ of size $\varepsilon$. The stable manifold of $q$ for $G$ is the set $W^{s}(q, G)=\left\{m: d\left(G^{k}(q), G^{k}(m)\right) \rightarrow 0\right.$ as $\left.k \rightarrow \infty\right\}$. Note that $W^{s}(q, G)=$ $\bigcup\left\{G^{-j}\left(W_{\varepsilon}^{s}\left(G^{j}(q), G\right)\right): j \geq 0\right\}$. The unstable manifold of $q$ for $G$ is the set $W^{u}(q, g)=\left\{m: d\left(G^{k}(q), G^{k}(m)\right) \rightarrow 0\right.$ as $\left.k \rightarrow-\infty\right\}$, and $W^{u}(q, G)=$ $\bigcup\left\{G^{j}\left(W_{\varepsilon}^{u}\left(G^{-j}(q), G\right)\right): j \geq 0\right\}$.

A closed set $\Lambda$ is called a hyperbolic basic set for $G$ if

(a) it is invariant for $G$; i.e., $G(\Lambda)=\Lambda$,

(b) it has a hyperbolic structure for $G$,

(c) there is a point $q$ in $\Lambda$ with a dense orbit; i.e., closure $(O(q))=\Lambda$,

(d) $\Lambda$ has a local product structure; i.e., if $\varepsilon>0$ is sufficiently small and $p$ and $q$ are in $\Lambda$, then $W_{\varepsilon}^{u}(p, G) \cap W_{\varepsilon}^{s}(q, G) \subset \Lambda$.

The stable manifold of $\Lambda$ is denoted by $W^{s}(\Lambda)$ and the unstable manifold of $\Lambda$ is denoted by $W^{u}(\Lambda)$.

Central to the theory of infinitely many coexisting sinks is the concept of persistent tangencies. A hyperbolic basic set $\Lambda$ for a diffeomorphism $F$ has persistent tangencies of stable and unstable manifolds (i.e., $\Lambda$ is a wild hyperbolic set) if for any diffeomorphism $H$ which is near $F$ in the $C^{2}$ topology, there are points $q_{1}$ and $q_{2}$ in $\Lambda(H)$ for which $W^{S}\left(q_{2}, H\right)$ has a finite order tangency with $W^{u}\left(q_{1}, H\right)$. 
Locally, $W^{s}(\Lambda)$ and $W^{u}(\Lambda)$ are products of a Cantor set and an interval. A Cantor set is a closed set which contains no interior points or isolated points. Newhouse [8] introduced the concept of the thickness (see below) of a Cantor set and using this concept developed conditions which insured that two Cantor sets cannot be separated when subjected to small perturbations. From this result, Newhouse concluded that there are hyperbolic basic sets for which $W^{S}(\Lambda)$ and $W^{u}(\Lambda)$ cannot be separated under small perturbations.

A Cantor set $A$ in the line is represented as the difference of an interval $A_{0}$ and an infinite collection $\left\{U_{j}\right\}$ of disjoint open subintervals (also known as gaps) contained in $A_{0}$. More precisely, $A=\bigcap_{i \geq 0} A_{i}$ where $A_{0}$ is the smallest interval containing $A$, and $A_{i}=A_{0}-\bigcup_{1 \geq j \geq i} U_{j}$. Such a sequence of sets $\left\{A_{i}\right\}$ is called a defining sequence of $A$.

Let $I_{i j}$ for $j=1,2$ be the two components of $A_{i}$ on either side of the gap $U_{i}$ and let $l(J)$ be the length of an interval $J$. The thickness of a defining sequence is defined by $\tau\left(\left\{A_{i}\right\}\right)=\inf \left\{l\left(I_{i j}\right) / l\left(U_{i}\right): i \geq 1\right.$, where $\left.j=1,2\right\}$.

The thickness of a Cantor set is defined by $\tau(A)=\sup \left\{\tau\left(\left\{A_{i}\right\}\right):\left\{A_{i}\right\}\right.$ is a defining sequence for $A\}$. Using this definition, Newhouse [8] proved the following lemma:

Lemma. Let $A^{1}$ and $A^{2}$ be two Cantor sets in $R$ such that $\tau\left(A^{1}\right) \tau\left(A^{2}\right)>1$. If $A^{1}$ is not contained in a gap of $A^{2}$ and $A^{2}$ is not contained in a gap of $A^{1}$, then $A^{1} \cap A^{2} \neq \varnothing$.

We can now define the thickness of a hyperbolic basic set. Let $\Lambda$ be a hyperbolic basic set for $F$. The stable thickness of $\Lambda, \tau^{s}(\Lambda)$, is defined by

$$
\tau^{s}(\Lambda)=\limsup _{\varepsilon \rightarrow 0}\left\{\tau\left(\gamma_{\varepsilon} \cap W^{s}(\Lambda)\right)\right\}
$$

where $\gamma$ is any $C^{1}$ arc transverse to the stable manifolds $W^{s}(\Lambda)$ at $q$, and $\gamma_{\varepsilon}$ is the arc of length $\varepsilon$ in $\gamma$ centered at $q$. The unstable thickness, $\tau^{u}(\Lambda)$, is defined in a similar manner. Newhouse [10] shows that $\tau^{s}(\Lambda)$ and $\tau^{u}(\Lambda)$ are independent of $q$ and $\gamma$, and therefore, are well defined.

\section{SINKS FROM TANGENCIES}

Theorem 1.1. Let $\left\{F_{t}\right\}$ be a fixed one-parameter family of $C^{\infty}$ diffeomorphisms in two dimensions. Assume that at $t_{0},\left\{F_{t}\right\}$ creates degenerate homoclinic tangencies of constant finite order for the periodic points $P_{t}$ of period $n$ with

$$
\left|\operatorname{det} D F_{t_{0}}^{n}\left(P_{t_{0}}\right)\right|<1 \quad\left(\left|\operatorname{det} D F_{t_{0}}^{n}\left(P_{t_{0}}\right)>1\right|\right)
$$

and that $F_{t}^{n}$ is $C^{\infty}$ linearizable in a neighborhood of $P_{t}$. Then, for $\varepsilon>0$, there exists a subinterval $\left[t_{1}, t_{2}\right] \subset\left[t_{0}-\varepsilon, t_{0}+\varepsilon\right]$ and a residual subset $J$ in $\left[t_{1}, t_{2}\right]$ such that for $t$ in $J, F_{t}$ has infinitely many sinks (sources).

The difference between Theorem 1.1 and the Main Result is that in Theorem 1.1 we do not allow the manifold $W^{u}\left(P_{i}, F_{t}\right)$ to unfold near the homoclinic 
tangency. By eliminating the possibility of $W^{u}\left(P_{t}, F_{t}\right)$ unfolding, we can prove that there is a hyperbolic invariant set with large stable thickness near the point of tangency. Although Theorem 1.1 assumes $\left|\operatorname{det} D F_{t}^{n}\left(P_{t}\right)\right|<1$ for $t=t_{0}$, we will present our proof assuming

$$
0<\operatorname{det} D F_{t_{0}}\left(P_{t_{0}}\right)<1
$$

and that the eigenvalues $\mu$ and $\lambda$ of $P_{t}$ are positive and satisfy the following conditions:

(a) $0<\mu \lambda<1$,

(b) $0<\mu<1$,

(c) $\lambda>1$.

The other cases are similar.

Proceeding in a fashion similar to Robinson [13], we assume that there is a neighborhood $U$ of $P_{t}$ and $C^{\infty}$ coordinates on $U$ so that $F_{t}(x, y)=$ $(\mu x, \lambda y)$. We will let $q_{0}$ be the point at which $W^{s}\left(P_{t}, F_{t}\right)$ and $W^{u}\left(P_{t}, F_{t}\right)$ have a tangency at $t=t_{0}$. We can assume that $q_{0}=\left(x_{0}, 0\right)$ is in $U$ on the local stable manifold of $P_{t}$ when $t=t_{0}$. In addition, we can choose $k$ large enough so that $U$ contains

$$
q_{1}=F_{t_{0}}^{-k}\left(q_{0}\right)=\left(0, y_{1}\right) .
$$

Next, we form boxes $B_{n}$ near $q_{0}$. Let $\delta_{1}^{u}, \delta_{1}^{s}, \delta_{0}^{u}, \delta_{0}^{s}$ be positive and define

$$
\begin{aligned}
& V_{0}=\left\{(x, y):\left|x-x_{0}\right| \leq \delta_{0}^{s}, 0 \leq\left(\operatorname{sign} y_{1}\right) y \leq \delta_{0}^{u}\right\}, \\
& V_{1}=\left\{(x, y):\left|y-y_{1}\right| \leq \delta_{1}^{u}, 0 \leq\left(\operatorname{sign} x_{0}\right) x \leq \delta_{1}^{s}\right\} .
\end{aligned}
$$

The deltas are chosen so that $V_{0} \cap F_{t}\left(V_{0}\right)=\varnothing, V_{1} \cap F_{t}\left(V_{1}\right)=\varnothing, \gamma_{t}^{s} \subset$ boundary $V_{0}$, and $\gamma_{t}^{u} \subset$ boundary $F_{t}^{k}\left(V_{1}\right)$. Here, $\gamma_{t}^{s} \subset W^{s}\left(P_{t}, F_{t}\right)$ and $\gamma_{t}^{u} \subset W^{u}\left(P_{t}, F_{t}\right)$ are continuously varying subarcs which satisfy the definition that $\left\{F_{t}\right\}$ creates odd order homoclinic intersections at $t_{0}$ near $q_{0}$.

$B_{n}$ is a horizontal strip near $q_{0}$ and is defined to be the first intersection of $F_{t}^{-m}\left(V_{1}\right)$ with $V_{0}$. Specifically, if we let $m=n-k$, then $B_{n}$ is defined to be:

$$
\begin{aligned}
B_{n} & =\text { component }\left(V_{0} \cap F_{t}^{-m}\left(V_{1}\right)\right) \\
& =\left\{(x, y):\left|x-x_{0}\right| \leq \delta_{1}^{s},\left|y-\lambda^{-m} y_{1}\right|=\lambda^{-m} \delta_{1}^{u}\right\} .
\end{aligned}
$$

A vertical strip near $q_{1}$ is formed when the map $F_{t}^{m}$ is applied to the box $B_{n}$. This vertical strip is given by

$$
F_{t}^{m}\left(B_{n}\right)=\left\{(x, y):\left|y-y_{1}\right| \leq \delta_{1}^{u},\left|x-\mu^{m} x_{0}\right| \leq \mu^{m} \delta_{0}^{s}\right\} .
$$

Composing $F_{t}^{k}$ with $F_{t}^{m}$, we have that $F_{t}^{n}=F_{t}^{k} \circ F_{t}^{m}\left(B_{n}\right)$ is a thin nonlinear box near $q_{0}$ parallel to $W^{u}\left(P_{t}\right)$. See Figure 1 .

Throughout this section we will assume that $\left\{F_{t}\right\}$ creates degenerate homoclinic tangencies of constant finite order. Abiding by this assumption, we will prove the following: 


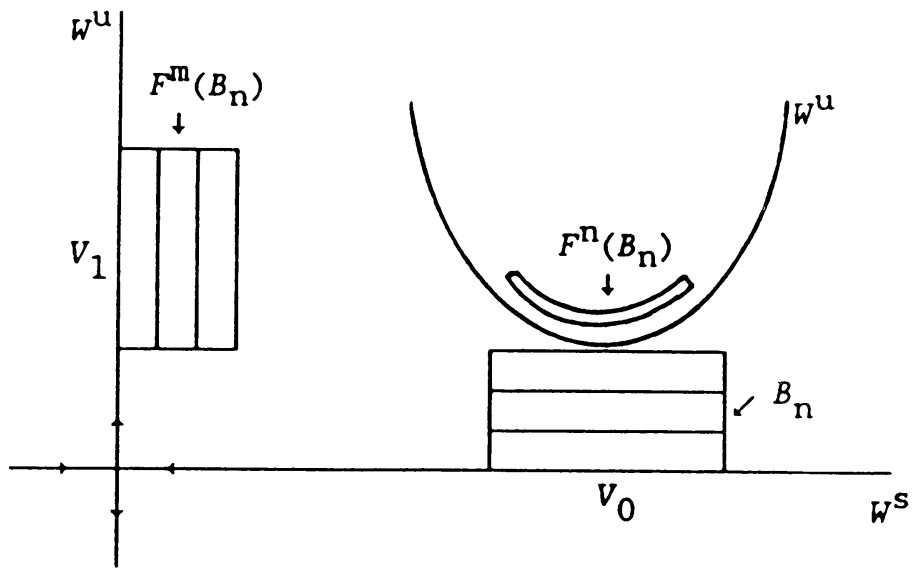

FigURE 1

Proposition 1.1. For suitably chosen $t>t_{n}+\varepsilon_{n}$, the hyperbolic basic set $\Lambda_{n}(t)$ created in $F_{t}^{n}\left(B_{n}\right) \cap B_{n}$ has arbitrarily large $\tau^{s}\left(\Lambda_{n}(t)\right)$.

In the proof of Proposition 1.1 a parameter value $t_{n}^{*}$ is chosen so that there exists a hyperbolic invariant set $\Lambda_{n}\left(t_{n}^{*}\right)$ in $B_{n}$ such that $\tau^{s}\left(\Lambda_{n}\left(t_{n}^{*}\right)\right)$ is arbitrarily large. The curve of $F_{t}^{n}\left(B_{n}\right)$ must protrude from the bottom of the box $B_{n}$ enough so that the maximal invariant set in $B_{n} \quad \Lambda_{n}(t)=\bigcap_{m} F_{t}^{m n}\left(B_{n}\right)$ has a hyperbolic structure. In order to do this, $t_{n}^{*}$ must be chosen large enough. On the other hand, $t_{n}^{*}$ must be chosen small enough so that $\Lambda_{n}\left(t_{n}^{*}\right)$ has large stable thickness. Unfortunately, for the choice $t=t_{n}^{*}$, there might not be any homoclinic tangencies between $W^{u}\left(\Lambda_{n}\left(t_{n}^{*}\right)\right)$ and $W^{s}\left(\Lambda_{n}\left(t_{n}^{*}\right)\right)$. This is due to the fact that the local extreme points of $W^{u}\left(\Lambda_{n}\left(t_{n}^{*}\right)\right)$ relative to $W^{s}\left(\Lambda_{n}\left(t_{n}^{*}\right)\right)$ lie away from $W^{s}\left(\Lambda_{n}\left(t_{n}^{*}\right)\right)$. For this reason, Theorem 1.1 does not follow directly from Proposition 1.1.

In Proposition 1.2, $t_{n}^{*}$ is decreased to $t_{n}^{* *}$ in order that the extreme points of $W^{u}\left(\Lambda_{n}\left(t_{n}^{* *}\right)\right)$ intersect with $W^{s}\left(\Lambda_{n}\left(t_{n}^{* *}\right)\right)$ thereby creating a persistent tangency between $W^{u}\left(\Lambda_{n}\left(t_{n}^{* *}\right)\right)$ and $W^{s}\left(\Lambda_{n}\left(t_{n}^{* *}\right)\right)$.

Proposition 1.2. For large enough $n$, there exists $t=t_{n}^{* *}$ such that

(a) $F_{t}^{n}$ has a hyperbolic basic set $\Lambda_{n}^{\prime}\left(t_{n}^{* *}\right) \subset B_{n}$,

(b) $F_{t}$ has a hyperbolic basic set $\Lambda\left(t_{n}^{* *}\right) \supset \Lambda_{n}^{\prime}\left(t_{n}^{* *}\right) \cup\left\{P_{t}\right\}$,

(c) $\tau^{s}\left(\Lambda\left(t_{n}^{* *}\right)\right) \tau^{u}\left(\Lambda\left(t_{n}^{* *}\right)\right)>1$,

(d) $\Lambda\left(t_{n}^{* *}\right)$ has a persistent tangency of $W^{s}\left(\Lambda\left(t_{n}^{* *}\right)\right)$ and $W^{u}\left(\Lambda\left(t_{n}^{* *}\right)\right)$.

As persistent tangencies are an open condition, Proposition 1.2 implies Proposition 1.3, and the proof of Theorem 1.1 follows from Proposition 1.3:

Proposition 1.3. Assume $\left\{F_{t}\right\}$ creates degenerate homoclinic tangencies of constant finite order at $t_{0}$ for the curve of periodic points $P_{t}$. Then given $\varepsilon>0$, 
there exists a subinterval $\left[t_{1}, t_{2}\right] \subset\left[t_{0}-\varepsilon, t_{0}+\varepsilon\right]$ such that for $t$ in $\left[t_{1}, t_{2}\right], F_{t}$ has a wild hyperbolic set containing the periodic point $P_{t}$.

Proof of Theorem 1.1. The hypothesis of Theorem 1.1 together with Proposition 1.3 implies that there exists an interval of parameter values $J_{0}=\left[t_{1}, t_{2}\right] \subset$ $\left[t_{0}-\varepsilon, t_{0}+\varepsilon\right]$ such that for each $t$ in $J_{0}$ there exists a homoclinic tangency of constant finite order of $W^{s}\left(\Lambda(t), F_{t}\right)$ and $W^{u}\left(\Lambda(t), F_{t}\right)$. Since $\Lambda(t)$ is a hyperbolic basic set, $\Lambda(t)$ has a dense orbit. For this reason, the manifold $W^{s}\left(P_{t}, F_{t}\right)$ is dense in $W^{s}\left(\Lambda(t), F_{t}\right)$, and the manifold $W^{u}\left(P_{t}, F_{t}\right)$ is dense in $W^{u}\left(\Lambda(t), F_{t}\right)$. By the definition of degenerate homoclinic tangencies of constant finite order, the tangency between $W^{s}\left(\Lambda(t), F_{t}\right)$ and $W^{u}\left(\Lambda(t), F_{t}\right)$ changes location as $t$ varies in $J_{0}$. Due to this, it follows that there is a dense set of parameter values $J^{\prime} \subset J_{0}$ such that for $t$ in $J^{\prime}$ the manifolds $W^{s}\left(P_{t}, F_{t}\right)$ and $W^{u}\left(P_{t}, F_{t}\right)$ have degenerate homoclinic tangencies of constant finite order.

Conforming to Robinson's theorem [13, p. 436], it follows that the set $J_{1} \subset$ $J_{0}$, for which $t$ in $J_{1}$ implies $F_{t}$ has at least one sink, is dense in $J_{0}$. In addition, $J_{1}$ is an open subset of $J_{0}$. Since $J^{\prime} \cap J_{1}$ is dense, repeating the argument proves that the set $J_{2}$, for which $t$ in $J_{2}$ implies $F_{t}$ has at least two sinks, is open and dense in $J_{0}$. By induction, the set $J_{k}$, such that $t$ in $J_{k}$ implies $F_{t}$ has at least $k$ sinks, is an open dense subset of $J_{0}$. As a result of this, $J=J_{\infty}=\bigcap\left\{J_{k}: k=1,2, \ldots\right\}$ is a residual subset of $J_{0}$; i.e., $J$ is a countable intersection of open dense sets. Moreover, for each $t$ in $J_{\infty}, F_{t}$ has infinitely many coexisting sinks.

We will now prove Proposition 1.1 using methods similar to those in Robinson [13]: Let $m=n-k$, and let $H_{t}=F_{t}^{k}$ be the map from $F_{t}^{m}\left(B_{n}\right) \subset V_{1}$ to $V_{0}$. Thus, $F_{t}^{n}$ from $B_{n}$ to $V_{0}$ is given by $F_{t}^{n}(x, y)=H_{t}\left(\lambda^{m} x, \mu^{m} y\right)$. For $t=t_{0}, H_{t}\left(0, y_{1}\right)=\left(x_{0}, 0\right)$ corresponds to the degenerate homoclinic tangency of constant finite order. Set

$$
\begin{aligned}
d_{1} & =\left|\frac{\partial H_{t}}{\partial y}\left(0, y_{1}\right)\right|, \quad d_{2}=\left|\frac{\partial H_{t}}{\partial x}\left(0, y_{1}\right)\right|, \\
y & =a\left(x-x_{n}(t)\right)^{r}+y_{n}(t), \quad r=2,4,6, \ldots \text { fixed },
\end{aligned}
$$

such that for $x^{\prime}$ fixed, $\left\{H_{t}\left(x^{\prime}, y\right):\left(x^{\prime}, y\right) \in F_{t}^{m}\left(B_{n}\right)\right\}$ is $C^{r}$ near $\{(x, y): y=$ $\left.a\left(x-x_{n}(t)\right)^{r}+y_{n}(t)\right\}$. Here, $\left(x_{n}(t), y_{n}(t)\right)$ is the point where $y$ has the smallest value on $F_{t}^{n}\left(B_{n}\right)$.

Let $g$ be the gap (distance) between the inside curve of $F_{t}^{n}\left(B_{n}\right)$ and the edge $A B$ of $B_{n}$. Then $g=\inf \left\{\sup \left\{d\left(F_{t}^{n}\left(x_{0}, y\right), B_{n}\right):\left(x_{0}, y\right) \in B_{n}, x_{0}\right.\right.$ fixed $\}$ : $x_{0}$ varies with $\left.\left(x_{0}, y^{\prime}\right) \in B_{n}\right\}$.

Let $g^{\prime}$ be the distance the lowest point on the bend is from the bottom of $B_{n}$. Then $g^{\prime}=\sup \left\{\sup \left\{d\left(F_{t}^{n}\left(x_{0}, y\right), B_{n}\right):\left(x_{0}, y\right) \in B_{n}, x_{0}\right.\right.$ fixed $\}: x_{0}$ varies with $\left.\left(x_{0}, y^{\prime}\right) \in B_{n}\right\}$. See Figure 2 . 


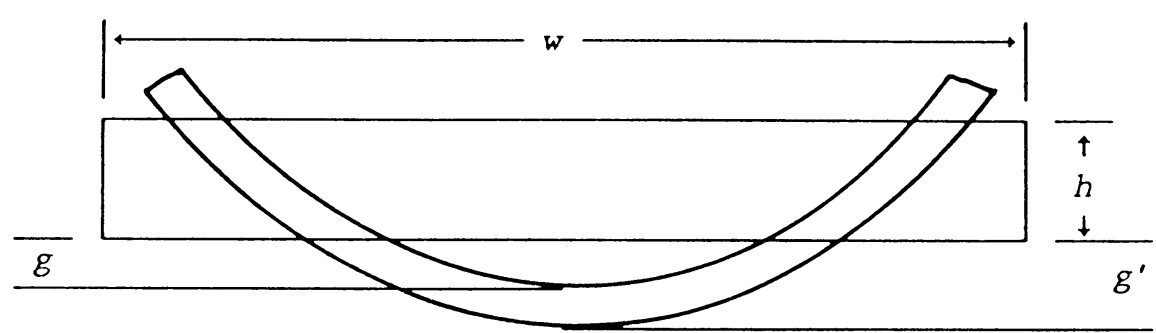

FigURE 2

The dimensions of the box $B_{n}$ and the bounds on $g^{\prime}$ and $g$ have to be chosen to satisfy four conditions:

(a) the width $w$ must be large enough so that $F_{t}^{n}\left(B_{n}\right)$ comes out the top of $B_{n}$ and not the sides,

(b) the height $h$ of $B_{n}$ must be large enough for $F_{t}^{n}\left(B_{n}\right)$ to stretch across $B_{n}$ from top to bottom and back to the top again,

(c) the bound on $g^{\prime}$ must be small enough to insure that $\tau^{s}\left(\Lambda_{n}(t)\right)$ is arbitrarily large,

(d) the bound on $g$ must be large enough to prove the set $\Lambda_{n}(t)$ has a hyperbolic structure.

In a series of lemmas below, we will show that the following choices for $w, h$, $g^{\prime}$, and $g$ satisfy conditions (a)-(d).

$$
\begin{gathered}
w=\left[\left(\frac{4^{r}+\alpha}{2 r}\right)^{r} \frac{\lambda^{-m}}{a d_{1}}\right]^{1 /(r-1)} ; \quad r=2,4,6, \ldots, \\
h=\frac{w \lambda^{-m}}{d_{1}-\alpha}=\left[\frac{4^{r}(1+\alpha) \lambda^{-m}}{2 r a^{1 / r} d_{1}}\right]^{r /(r-1)}, \\
g^{\prime} \leq \lambda^{-m} \beta A^{2} w=\left(d_{1}-\alpha\right) \beta A^{2} h, \\
g \geq a^{-1 /(r-1)} A^{r /(r-1)} r^{-r /(r-1)}\left(\mu \lambda^{-1}\right)^{m r / 2(r-1)}
\end{gathered}
$$

where $\alpha$ is a small unspecified constant. $\beta=\beta(m)$ is a small constant dependent on $m$ such that $\beta \rightarrow 0$ as $m \rightarrow 0$. In addition, $A$ is chosen such that $A^{2}>\eta\left(1-\eta^{-1}\right)^{-1}$ and $A^{2}$ is bounded, where $\eta$ is a constant such that $1<\eta<2^{1 / 4}$. First we shall show that the choice of the bounds of $g^{\prime}$ and $g$ are such that the gap $g$ is actually smaller than the gap $g^{\prime}$.

Lemma 1.1. $g^{\prime}>g$ for $m$ large and $\beta$ small.

Proof. The bound on $g^{\prime}$ is $\lambda^{-m} \beta A^{2} w$ and the bound on $g$ is

$$
a^{-1 /(r-1)} A^{r /(r-1)} r^{-r /(r-1)}\left(\mu \lambda^{-1}\right)^{m r / 2(r-1)} .
$$


To show that $g<g^{\prime}$, it is sufficient to show that the following inequality holds:

$$
\begin{gathered}
a^{-1 /(r-1)} A^{r /(r-1)} r^{-r /(r-1)}\left(\mu \lambda^{-1}\right)^{m r / 2(r-1)}<A^{2} \beta \lambda^{-m}\left[\left(\frac{4^{r}+\alpha}{2 r}\right)^{r} \frac{\lambda^{-m}}{a d_{1}}\right]^{1 /(r-1)}, \\
\left(\mu \lambda^{-1}\right)^{m r / 2(r-1)} \lambda^{m} \lambda^{m /(j-1)}<A^{2} A^{-r /(r-1)} r^{r /(r-1)} \beta d_{1}^{-1 /(r-1)}\left(\frac{4^{r}+\alpha}{2 r}\right)^{r /(r-1)}, \\
\left(\mu \lambda^{-1}\right)^{m r / 2(r-1)} \lambda^{m+m /(j-1)}<A^{2} A^{-r /(r-1)} r^{r /(r-1)} \beta d_{1}^{-1 /(r-1)}\left(\frac{4^{r}+\alpha}{2 r}\right)^{r /(r-1)}, \\
\left(\mu \lambda^{-1}\right)^{m r / 2(r-1)} \lambda^{m j /(j-1)}<A^{2} A^{-r /(r-1)} r^{r /(r-1)} \beta d_{1}^{-1 /(r-1)}\left(\frac{4^{r}+\alpha}{2 r}\right)^{r /(r-1)}, \\
(\mu \lambda)^{m r / 2(r-1)}<A^{2} A^{-r /(r-1)} r^{r /(r-1)} \beta d_{1}^{-1 /(r-1)}\left(\frac{4^{r}+\alpha}{2 r}\right)^{r /(r-1)} .
\end{gathered}
$$

In the final inequality, the left side of the equation approaches zero as $m$ approaches infinity because $\mu \lambda<1$; therefore, we can choose $\beta$ small enough to satisfy the inequality. Consequently, $g^{\prime}>g$.

Lemma 1.2. The width $w$ is large enough so that $F_{t}^{n}\left(B_{n}\right)$ protrudes from the top of $B_{n}$ and not the sides.

Proof. To show that $F_{t}^{n}\left(B_{n}\right)$ protrudes from the top of $B_{n}$ rather than the sides, it is sufficient to show that $a(w / 2)^{r}$ is larger than

$$
h+g^{\prime}=w \lambda^{-m}\left[\left(d_{1}-\alpha\right)^{-1}+\beta A^{2}\right] .
$$

That is, $a(w / 2)^{r} \geq w \lambda^{-m}\left[\left(d_{1}-\alpha\right)^{-1}+\beta A^{2}\right]$.

Since $\beta \rightarrow 0$ as $m \rightarrow 0$, and $A^{2}$ is bounded,

$$
w^{r-1} \geq a^{-1} 2^{r} \lambda^{-m}\left[\left(d_{1}-\alpha\right)^{-1}\right], \quad w \geq\left[\frac{2^{r} \lambda^{-m}}{a\left(d_{1}-\alpha\right)}\right]^{1 /(r-1)} .
$$

Thus, the choice,

is adequate.

$$
w=\left[\left(\frac{4^{r}+\alpha}{2 r}\right)^{r} \frac{\lambda^{-m}}{a d_{1}}\right]^{1 /(r-1)}
$$

Lemma 1.3. The choice for the height $h$ of $B_{n}$ is large enough for $F_{t}^{n}\left(B_{n}\right)$ to stretch across $B_{n}$ from top to bottom and back to the top again.

Proof. For $x^{\prime}$ fixed, on either side of the local minimum of $W^{u}\left(P_{t}, F_{t}\right)$, the curve $F_{t}^{n}\left\{\left(x^{\prime}, y\right) \in B_{n}\right\}$ is monotone; therefore its length is less than the sum of the change in the $x$ value plus the change in the $y$ value. In order to show that $F_{t}^{n}\left(B_{n}\right)$ can stretch across $B_{n}$, it suffices to show that

$$
\begin{gathered}
\left(d_{1}-\alpha\right) \lambda^{m} h \geq 2(w / 2)+2\left(h+g^{\prime}\right)=w+2 h+2 g^{\prime}, \\
{\left[\left(d_{1}-\alpha\right) \lambda^{m}-2\right] h \geq w\left[1+2 \lambda^{-m} \beta A^{2}\right],} \\
h \geq w\left[\left(d_{1}-\alpha\right) \lambda^{m}-2\right]^{-1}\left[1+2 \lambda^{-m} \beta A^{2}\right] .
\end{gathered}
$$


Thus, the value,

suffices.

$$
h=\frac{w \lambda^{-m}}{d_{1}-\alpha}
$$

Lemma 1.4. The bound on $g^{\prime}$ is small enough to insure that $\tau^{s}\left(\Lambda_{n}(t)\right)$ is arbitrarily large.

Proof. We will show that for $x^{\prime}$ fixed, the length of a component of

$$
F_{t}^{n}\left\{\left(x^{\prime}, y\right):\left(x^{\prime}, y\right) \in B_{n}\right\} \cap B_{n}
$$

is greater than the length of the gap $F_{t}^{n}\left\{\left(x^{\prime}, y\right) \in B_{n}\right\}-B_{n}$.

The length of a component of $F_{t}^{n}\left\{\left(x^{\prime}, y\right) \in B_{n}\right\} \cap B_{n}$ is greater than the change in its $x$ coordinate; i.e.,

$$
(h / a)^{1 / r}-\left(g^{\prime} / a\right)^{1 / r} \geq w^{1 / r} \lambda^{-m / r}\left(d_{1}-\alpha\right)^{-1 / r}-\left(\lambda^{-m} \beta A^{2} w a^{-1}\right)^{1 / r} .
$$

The length of the gap is less than the sum of the change in its $x$ coordinate plus the change in its $y$ coordinate; i.e.,

$$
g^{\prime}+\left(g^{\prime} / a\right)^{1 / r} \leq \lambda^{-m} \beta A^{2} w+\left(\lambda^{-m} \beta A^{2} w a^{-1}\right)^{1 / r} .
$$

The ratio of the length of a component to the length of the gap is greater than

$$
\frac{w^{1 / r} \lambda^{-m / r}\left(d_{1}-\alpha\right)^{-1 / r}-\left(\lambda^{-m} \beta A^{2} w a^{-1}\right)^{1 / r}}{\lambda^{-m} \beta A^{2} w+\left(\lambda^{-m} \beta A^{2} w a^{-1}\right)^{1 / r}},
$$

which is greater than

$$
\frac{w^{1 / r} \lambda^{-m / r}\left(a d_{1}\right)^{-1 / r}}{\left(\lambda^{-m} \beta A^{2} w a^{-1}\right)^{1 / r}}
$$

which equals $\left(\beta A^{2} d_{1}\right)^{-1 / r}$. Therefore, by choosing $\beta$ small enough compared to $A^{2}$ ( $A^{2}$ is bounded), we can assure that $\tau^{s}\left(\Lambda_{n}(t)\right)$ is arbitrarily large. (See Newhouse [10, pp. 134-136], to see how the above estimate implies large thickness.)

To show that $\Lambda_{n}(t)$ has a hyperbolic structure, it is sufficient to find invariant unstable sectors $S(z) \subset T_{z} M$ for $z$ in $F_{t}^{n}\left(B_{n}\right) \cap B_{n}$ which are invariant and expanded by $D F_{t}^{k n}(z)$ for large $k$. Not all of the vectors in the unstable sectors, however, are expanded for $k=1$; there is a power $j=k(z) n$ dependent on the point $z$ which expands vectors. Using a compactness argument, it can be shown that there is one power $k n$ which works for all points in $\Lambda_{n}(t)$ thereby proving the existence of an expanding invariant subbundle.

First, it is necessary to define the sectors $S(z)$. For $z=(x, y)$ in $F_{t}^{n}\left(B_{n}\right)$ $\cap B_{n}$, and $z_{-1}=F_{t}^{-n}(z)$, the slope of $\left(\partial F_{t}^{n} / \partial y\right)\left(z_{-1}\right)$ is approximately $r a\left(x-x_{n}(t)\right)^{r-1}$. Define the functions $\xi(z)$ and $\xi^{+}(z)$ by

$$
\begin{gathered}
\xi(z)=r a\left(x-x_{n}(t)\right)^{r-1}, \\
\xi^{+}(z)=|\xi(z)|=r a^{1 / r}\left(y-y_{n}(t)\right)^{(r-1) / r} .
\end{gathered}
$$


The sectors, $S(z)$, are defined by

$$
S(z)=\left\{\nu=\left(\nu_{1}, \nu_{2}\right) \in T_{z} M: \eta^{-1} \leq \frac{\nu_{2}}{\nu_{1} \xi(z)} \leq \eta\right\} .
$$

Therefore, for $\nu$ in $S(z)$, the slope of $\nu$ lies between $\eta^{-1} \xi(z)$ and $\eta \xi(z)$. This allows $\nu$ to have either positive or negative slope depending on the sign of $\xi(z)$.

If $\nu=\left(\nu_{1}, \nu_{2}\right) \in S(z)$, then $\nu^{\prime}=\left(\nu_{1}^{\prime}, \nu_{2}^{\prime}\right)$ is defined to be the image of $\nu$ under $D F_{t}^{m}(z)$; i.e., $\nu^{\prime}=D F_{t}^{m}(z)\left(\nu_{1}, \nu_{2}\right)=\left(\mu^{m} \nu_{1}, \lambda^{m} \nu_{2}\right)$. Also, we define $\nu^{\prime \prime}=\left(\nu_{1}^{\prime \prime}, \nu_{2}^{\prime \prime}\right)=D F_{t}^{n}(z)\left(\nu_{1}, \nu_{2}\right)=D H_{t}\left(F_{t}^{m}(z)\right)\left(\nu_{1}^{\prime}, \nu_{2}^{\prime}\right)$.

Lemma 1.5. The sectors $S(z)$ are invariant under $D F_{t}^{n}$; i.e., if $z$ and $F_{t}^{n}(z)$ are in $B_{n} \cap F_{t}^{n}\left(B_{n}\right)$, then $D F_{t}^{n}(z) S(z) \subset S\left(F_{t}^{n}(z)\right)$.

Proof. Recall the choice of the bound on $g$

$$
g \geq a^{-1 /(r-1)} A^{r /(r-1)} r^{-r /(r-1)}\left(\mu \lambda^{-1}\right)^{m r / 2(r-1)} .
$$

Then,

$$
\begin{aligned}
\xi^{+}(z) & =r a^{1 / r}\left(y-y_{n}(t)\right)^{(r-1) / r} \geq r a^{1 / r} g^{(r-1) / r} \\
& =r a^{1 / r} a^{-1 / r} A r^{-1}\left(\mu \lambda^{-1}\right)^{m / 2}=A\left(\mu \lambda^{-1}\right)^{m / 2} .
\end{aligned}
$$

Denote the slope of $\nu$ by $s(\nu)$ where $\nu \in S(z)$. Then for any $z$ in the box $B_{n}$ we have that $s(\nu) \geq \eta^{-1} A\left(\mu \lambda^{-1}\right)^{m / 2}$. We also have that

$$
s\left(\nu^{\prime}\right)=\left(\lambda \mu^{-1}\right)^{m} s(\nu) \geq\left(\lambda \mu^{-1}\right)^{m} \eta^{-1} A\left(\mu \lambda^{-1}\right)^{m / 2}=\eta^{-1} A\left(\lambda \mu^{-1}\right)^{m / 2} .
$$

We require that the angle between $\nu^{\prime}$ and $W^{u}\left(P_{t}, F_{t}\right)$ is small enough to insure that the image of $\nu^{\prime}$ (i.e., $\left.\nu^{\prime \prime}\right)$ lies in $S\left(F_{t}^{n}(z)\right)$ :

$$
\frac{1}{s\left(\nu^{\prime}\right)}<\xi^{+} \frac{1-\eta^{-1}}{1+\eta^{-1}\left(\xi^{+}\right)^{2}}=\xi^{+}\left[\left(1-\eta^{-1}\right)+O(m)\right] .
$$

Employing the preceding inequality together with the fact that $\left[s\left(\nu^{\prime}\right)\right]^{-1}<$ $\eta\left(\mu \lambda^{-1}\right)^{m / 2} A^{-1}$, we can show that $\nu^{\prime \prime}$ lies in $S\left(F_{t}^{n}(z)\right)$ if

$$
\eta\left(\mu \lambda^{-1}\right)^{m / 2} A^{-1}<\left(\mu \lambda^{-1}\right)^{m / 2} A\left[\left(1-\eta^{-1}\right)+O(m)\right] ;
$$

that is, if $A^{2}>\eta\left[\left(1-\eta^{-1}\right)+O(m)\right]^{-1}$. Therefore, if $A$ is relatively large, then the sectors $S(z)$ are invariant under $D F_{t}^{n}$.

Next, we show that for $z$ bounded away from the bend at the bottom, the vectors in $S(z)$ are immediately expanded.

Lemma 1.6. If $z=(x, y)$ is in $B_{n}$ with $y-y_{n}(t)=\gamma h$ and $\nu$ is in $S(z)$, then

$$
\left\|D F_{t}^{n}(z) \nu\right\| \geq \eta^{-1}(1-\alpha) 2^{-1} 4^{r} \gamma^{(r-1) / r}\|\nu\| \text {. }
$$

Furthermore. if $\gamma \geq 4^{-r}$, then $\left\|D F_{t}^{n}(z) \nu\right\|>\|\nu\|$. 
Proof. Let $\nu^{\prime \prime}=D F_{t}^{n}(z) \nu$. Then

$$
\begin{aligned}
\left\|\nu^{\prime \prime}\right\| & \geq d_{1}(1-\alpha) \lambda^{m} \eta^{-1} \xi^{+}(z)\|\nu\| \\
& \geq d_{1}(1-\alpha) \lambda^{m} \eta^{-1} r a^{1 / r}(\gamma h)^{(r-1) / r}\|\nu\| \\
& \geq d_{1}(1-\alpha) \lambda^{m} \eta^{-1} r a^{1 / r} \gamma^{(r-1) / r} h^{(r-1) / r}\|\nu\| .
\end{aligned}
$$

Hence, if $\gamma>4^{-r}$, then

$$
\begin{aligned}
\left\|\nu^{\prime \prime}\right\| & \geq \eta^{-1}(1-\alpha) 4^{1-r} 4^{r} 2^{-1}\|\nu\| \\
& \geq \eta^{-1}(1-\alpha) 2\|\nu\|>1
\end{aligned}
$$

since $1<\eta<2^{1 / 4}$.

We have shown in Lemma 1.6 that vectors belonging to certain sectors $S(z)$ are not expanded under $D F_{t}^{n}(z)$. In fact, if $z=(x, y)$, and $y$ is such that $y-y_{n}(t)<4^{-r} h$, then the derivatives $D F_{t}^{n}(z)$ contracts vectors in $S(z)$. The points $z$ for which $\nu$ in $S(z)$ were not initially expanded, i.e., those near the bottom of $B_{n}$, are mapped close to the top of $B_{n}$ under $F_{t}^{n}$. The next few iterates of $z$ remain where vectors are expanded; that is, near the top of $B_{n}$.

We will denote the $y$ value on the bottom of $B_{n}$ by $y_{0}$, and we will let the top half of the box $B_{n}$ be denoted by $H_{1}$; that is, $H_{1}=\left\{z \in B_{n}: y-y_{0} \geq h / 2\right\}$. Moreover, for $z=(x, y)$ with the condition that $y-y_{0} \leq 4^{-r} h$, we denote $k=k(z)$ to be the unique integer with the property that $F_{t}^{n j}(z) \in H_{1}$ when $1 \leq$ $j \leq k(z)$ and $F_{t}^{n(k+1)}(z) \notin H_{1}$. Because of the way that $k$ has been chosen, the images of the points $z$ [i.e., $z_{j}=F_{t}^{n j}(z)$ ] are such that $y_{j}-y_{n}(t) \geq h / 2$, and therefore, $D F_{t}^{n}\left(z_{j}\right)$ will expand the vectors in $S\left(z_{j}\right)$.

Lemma 1.7 shows that the total effect of $D F_{t}^{n(k+1)}(z)$ is to expand the vectors in $S(z)$ :

Lemma 1.7. If $y-y_{0} \leq 4^{-r} h$, then for $k=k(z)$ as defined in the previous paragraph, and $\nu$ in $S(z),\left\|D F_{t}^{n k+n}(z) \nu\right\|>\|\nu\|$.

Proof. Let $z=(x, y)$ be a point in $B_{n}$ having the property $y-y_{0} \leq \gamma h$. In order to estimate the number of iterates $z$ remains in $H_{1}$ [i.e., in order to estimate $k(z)$ ], it is vital to show that $z_{1}=\left(x_{1}, y_{1}\right)=F_{t}^{n}(z)$ is close to the top of the box $B_{n}$. It is also necessary to compare the maximal and minimal stretches of vectors in $S(\zeta)$ where $\zeta$ is in $H_{1}$.

Note that the absolute value of the partial derivative $\partial F_{t}^{n} / \partial y$ is less than $(1+\alpha) d_{1} \lambda^{m}$. Furthermore, the slope of $F_{t}^{n}\left(B_{n}\right)$ is always less than the value of the slope $s$ at the top of the box $B_{n}$. Consequently, we have that

$$
\begin{aligned}
s & \leq r a^{1 / r}\left(h+g^{\prime}\right)^{(r-1) / r}<r a^{1 / r} h^{(r-1) / r}(1+\alpha) \\
& <r a^{1 / r} \frac{4^{r}(1+\alpha) \lambda^{-m}}{2 r a^{1 / r} d_{1}}<4^{r} 2^{-1}(1+\alpha) \lambda^{-m} d_{1}^{-1},
\end{aligned}
$$

because $g^{\prime} / h$ is small. 
Let $y_{\text {top }}$ be the value of $y$ on the top of $B_{n}$; then

$$
y_{\text {top }}-y_{1} \leq \gamma h(1+\alpha) d_{1} \lambda^{m} s \leq \gamma h(1+\alpha) 4^{r} 2^{-1}<h / 2,
$$

because $\gamma \leq 4^{-r}$.

Let $\xi_{\min }$ be the minimal value of $\xi^{+}(\zeta)$ for $\zeta$ in $H_{1}$, and let $\xi_{\max }$ be the maximal value of $\xi^{+}(\zeta)$ for $\zeta$ in $H_{1}$; then

$$
\begin{aligned}
\xi_{\min } & \geq r a^{1 / r}(h / 2)^{(r-1) / r} \geq r a^{1 / r} 2^{(r-1) / r} h^{(r-1) / r} \\
& \geq r a^{1 / r} 2^{(r-1) / r} \frac{4^{r}(1+\alpha) \lambda^{-m}}{2 r a^{1 / r} d_{1}} \geq 4^{r} 2^{-1} 2^{(r-1) / r} d_{1}^{-1} \lambda^{-m}
\end{aligned}
$$

and

$$
\xi_{\max } \leq 4^{r} 2^{-1}(1+\alpha) \lambda^{-m} d_{1}^{-1} .
$$

Let $\lambda_{\min }$ be the minimal stretch of vectors in $S(\zeta)$ for $\zeta$ in $H_{1}$, and let $\lambda_{\max }$ be the maximal stretch of vectors in $S(\zeta)$ for $\zeta$ in $H_{1}$; then

$$
\begin{gathered}
\lambda_{\min } \geq \lambda^{m}\left(d_{1}-\alpha\right) \eta^{-1} \xi_{\min } \geq \eta^{-1}(1-\alpha) 2^{(r-1) / r} 4^{r} 2^{-1}, \\
\lambda_{\max } \leq \lambda^{m}\left(d_{1}+\alpha\right) \eta \xi_{\max } \leq \eta(1+\alpha) 4^{r} 2^{-1} .
\end{gathered}
$$

If we raise the preceding inequality for $\lambda_{\max }$ to the $(r-1) / r$ power, we can obtain a bound on $\lambda_{\max }^{(r-1) / r}$; i.e.,

$$
\lambda_{\max }^{(r-1) / r} \leq \eta(1+\alpha) 4^{r-1} 2^{(r-1) / r} .
$$

Using this bound together with the inequality for $\lambda_{\min }$, we obtain the following inequality:

$$
\begin{aligned}
\frac{\lambda_{\min }}{\lambda_{\max }^{(r-1) / r}} & \geq \frac{\eta^{-1}(1-\alpha) 2^{(r-1) / r} 4^{r} 2^{-1}}{\eta(1+\alpha) 4^{r-1} 2^{(r-1) / r}} \\
& \geq 2 \eta^{-2}(1-\alpha) \geq 2^{1 / 2}(1-\alpha) \geq \delta>1
\end{aligned}
$$

since $1<\eta<2^{1 / 4}$. We then have that,

$$
\lambda_{\min } \geq \delta \lambda_{\max }^{(r-1) / r}
$$

and

$$
\lambda_{\min }^{k} \geq \delta \lambda_{\max }^{k(r-1) / r}
$$

due to the fact that $k=k(z) \geq 1$.

The distance between the end of the strip $F_{t}^{n}\left(B_{n}\right)$ and the point $z_{1}$ is less than $\gamma h(1+\alpha) d_{1} \lambda^{m}$. Also, $F_{t}^{n}$ stretches lengths along $F_{t}^{n}\left(B_{n}\right)$ by a factor less than $\lambda_{\max }$. Therefore, it follows that the value $k=k(z)$ satisfies,

$$
\begin{gathered}
\gamma h(1+\alpha) d_{1} \lambda^{m} \lambda_{\max }^{k} s \geq h / 2, \\
\gamma h 4^{r} 2^{-1}(1+\alpha) \lambda_{\max }^{k} \geq h / 2,
\end{gathered}
$$


which implies that

$$
\lambda_{\max }^{k} \geq(1-\alpha) 4^{-r} \gamma^{-1}
$$

This bound on $\lambda_{\max }^{k}$ together with

$$
\lambda_{\min }^{k} \geq \delta \lambda_{\max }^{k(r-1) / r}
$$

gives us the following estimate of $\lambda_{\min }^{k}$,

$$
\lambda_{\min }^{k} \geq \delta(1-\alpha) 4^{1-r} \gamma^{(r-1) / r} .
$$

By reason of this estimate, if $z$ is a point in $B_{n}$ which satisfies $y-y_{n}(t) \leq$ $h 4^{-r}$, then all the vectors $\nu$ contained in the sector $S(z)$ satisfy the following inequalities:

$$
\begin{aligned}
& \left\|D F_{t}^{n k+n}(z) \nu\right\| \cdot\|\nu\|^{-1} \geq \lambda^{m}\left(d_{1}-\alpha\right) \eta^{-1} \xi^{+}(z) \lambda_{\min }^{k} \\
& \quad \geq(1-\alpha) \lambda^{m} d_{1} \eta^{-1} r a^{1 / r} \gamma^{(r-1) / r} h^{(r-1) / r}(1-\alpha) \delta 4^{1-r} \gamma^{(1-r) / r} \\
& \quad \geq(1-\alpha) \lambda^{m} d_{1} \eta^{-1} r a^{1 / r} \delta 4^{1-r} \frac{4^{r}(1+\alpha) \lambda^{-m}}{2 r a^{1 / r} d_{1}} \\
& \quad \geq 2(1-\alpha) \eta^{-1} \delta>1
\end{aligned}
$$

for the reason that $1<\eta<2^{1 / 4}$ and $\delta>1$.

Lemma 1.8 [2]. There exist constants $C>0$ and $\lambda_{u}>1$ such that for all $\nu$ in $S(z)$ [ with $z$ in $\Lambda_{n}(t)$ ], we have that $\left\|D F_{t}^{n j}(z) \nu\right\| \geq C \lambda_{u}^{j}\|\nu\|$, for all $j \geq 0$. Proof. For each $z$ in $\Lambda_{n}(t)$, there exists an integer $k=k(z)$ such that

$$
\left\|D F_{t}^{n k}(z) \nu\right\| \cdot\|\nu\|^{-1}>1
$$

for all vectors $\nu$ in $S(z)$. Therefore, for each $z$ in $\Lambda_{n}(t)$, there is a constant $\lambda_{z}>1$ which has the property $\left\|D F_{t}^{n k}(z) \nu\right\|>\lambda_{z}^{n k}\|\nu\|$. Moreover, for each $z$ in $\Lambda_{n}(t)$, there is a neighborhood $U(z)$ of $z$ such that for all $z^{\prime} \in \operatorname{cl} U(z)$, the vectors $\nu^{\prime} \in S\left(z^{\prime}\right)$ are stretched for the same iterate $k=k(z)$; i.e., $\left\|D F_{t}^{n k}\left(z^{\prime}\right) \nu^{\prime}\right\|>\lambda_{z}^{n k}\left\|\nu^{\prime}\right\|$.

By compactness, we can choose a finite number of points $z_{1}, \ldots, z_{p}$ such that $\Lambda_{n}(t) \subset U\left(z_{1}\right) \cup \cdots \cup U\left(z_{p}\right)$. We define $\lambda_{u}$ to be the infimum of the $\lambda_{z_{i}}$; i.e.,

$$
\lambda_{u}=\inf \left\{\lambda_{z_{i}}\right\}, \quad 1 \leq i \leq p .
$$

Because of the way $\lambda_{u}$ is defined, we have that

$$
\left\|D F_{t}^{n k\left(z_{i}\right)}\left(z^{\prime}\right) \nu\right\|>\lambda_{u}^{n k\left(z_{i}\right)}\|\nu\|
$$

for all $z^{\prime}$ contained in $\operatorname{cl} U\left(z_{i}\right), 1 \leq i \leq p$.

Let $z$ be an arbitrary point in $\Lambda_{n}(t)$ and choose a sequence of integers $i(1), i(2), \ldots$ as follows. Choose $i(1)$ so that $z$ is contained in the set $U\left(z_{i(1)}\right)$. We suppose that $i(1), \ldots, i(q)$ have been chosen, and we set $\varphi(q)=$ $k\left(z_{i(1)}\right)+\cdots+k\left(z_{i(q)}\right)$. Now we choose $i(q+1)$ such that $F_{t}^{-\varphi(q)}(z) \in U\left(z_{i(q+1)}\right)$. 
If we let $j$ be any positive integer, we can write $j=\varphi(q)+r$ for some $q$, where $0 \leq r \leq \max k\left(z_{i}\right)$. It then follows that

$$
\begin{aligned}
\left\|D F_{t}^{n j}(z) \nu\right\| & =\left\|D F_{t}^{n r}\left(F_{t}^{-\varphi(q)}(z)\right) \circ D F_{t}^{k\left(z_{i(q)}\right)}\left(F_{t}^{-\varphi(q-1)}(z)\right) \circ \cdots \circ D F_{t}^{\left(z_{i(1)}\right)}(z) \nu\right\| \\
& \geq\left\|D F_{t}^{n r}(z) \nu\right\| \lambda_{u}^{n k\left(z_{1(q)}\right)} \cdots \lambda_{u}^{n k\left(z_{i(1)}\right)}\|\nu\| \geq C \lambda_{u}^{n j}\|\nu\|,
\end{aligned}
$$

where

$$
C=\frac{\inf \left\|D F_{t}^{n r}(z)\right\|}{\lambda_{u}^{n r}}
$$

and the infimum is taken over all $z$ in $\Lambda_{n}(t)$ and all $r$, such that $0 \leq r \leq$ $\max k(z)$.

Lemma 1.9. The bound on $g$ is large enough so that the maximal invariant set $\Lambda_{n}(t)$ has a hyperbolic structure.

Proof. According to Lemma 1.5, the sector $S(z)$ is mapped inside of the sector $S\left(F_{t}^{n}(z)\right)$. Because of this, there is an invariant bundle $E_{z}^{u}$ for $z$ in $\Lambda_{n}(t)$. Lemma 1.6 together with Lemma 1.7 shows that the vectors $\nu$ contained in $E_{z}^{u}$ are eventually expanded. In addition, Lemma 1.8 proves that there are constants $C>0$ and $\lambda_{u}>1$ with the property that for each $\nu$ in $E_{z}^{u}$ and for each $j \geq 0$ we have $\left\|D F_{t}^{n j}(z) \nu\right\| \geq C \lambda_{u}^{j}\|\nu\|$.

In order to show that there is a contracting invariant bundle $E_{z}^{s}$, let $S^{*}(z)$ be the complementary bundle to $S(z) . S^{*}(z)$ is overflowing by $D F_{t}^{n}(z)$, and due to this, the bundle $S^{*}(z)$ is invariant under $D F_{t}^{-n}(z)$; i.e., $D F_{t}^{-n}(z) S^{*}(z) \subset$ $S^{*}\left(F_{t}^{-n}(z)\right)$. As a result of this, there is an invariant bundle $E_{z}^{s}$ associated to each $z$ in $\Lambda_{n}(t)$. Since $\operatorname{det}\left(D F_{t}^{n}(z)\right)<1$ and $D F_{t}^{n}(z)$ expands vectors in the unstable invariant bundle $E_{z}^{u}, D F_{t}^{n}(z)$ must contract vectors in the complementary invariant bundle $E_{z}^{s}$. Therefore, $\Lambda_{n}(t)$ has a hyperbolic structure.

Proof of Proposition 1.1. In Lemma 1.2 we proved $F_{t}^{n}\left(B_{n}\right)$ protrudes from the top of $B_{n}$ and not the sides. In Lemma 1.3 we proved $F_{t}^{n}\left(B_{n}\right)$ is long enough to stretch across $B_{n}$ from the top to the bottom and back to the top again. These facts together with the choice of the gap $g$, imply that the set $\Lambda_{n}(t)$ is conjugate to a shift of two symbols; and therefore, $\Lambda_{n}(t)$ has a dense orbit. $\Lambda_{n}(t)$ has a hyperbolic structure by Lemma 1.9; hence, $\Lambda_{n}(t)$ is a hyperbolic basic set. Furthermore, in Lemma 1.4 we proved $\tau^{s}\left(\Lambda_{n}(t)\right)$ is arbitrarily large. Therefore, the proof of Proposition 1.1 is complete.

We will now turn our attention to Proposition 1.2. The main idea behind the proof of Proposition 1.2 is to reduce the parameter value, as determined in Proposition 1.1, to a nearby parameter value where there are persistent tangencies.

Proof of Proposition 1.2. We assume that $P_{t}$ is an element of the hyperbolic set $\Lambda_{1}\left(t_{1}\right)$. This is justified by the fact that there is a parameter value $t=t_{1}$ near 
$t_{0}$ such that $W^{u}\left(P_{t}, F_{t}\right)$ has points of transverse intersection with $W^{s}\left(P_{t}, F_{t}\right)$ and other points where there is a degenerate homoclinic tangency of constant finite order. (See references [3], [4], [5] or [10] for more information.)

We choose a number $T$ such that $T \tau^{u}\left(\Lambda_{1}\left(t_{1}\right)\right)>4$. The thickness of a hyperbolic set varies continuously for $C^{2}$ changes of $F_{t}$ [10]. Therefore, there is an interval $I$ of parameter values which contains $t_{1}$ such that for each $t$ in $I$, we have that $T \tau^{u}\left(\Lambda_{1}(t)\right)>2$. According to Proposition 1.1, if $n$ is large enough, then there exists a value of $t$ in $I, t_{n}$, and a corresponding hyperbolic set belonging to $F_{t}^{n}, \Lambda_{n}\left(t_{n}\right) \subset B_{n}$, for which $\tau^{s}\left(\Lambda_{n}\left(t_{n}\right)\right)>T$.

Let $p_{n}\left(t_{n}\right)$ be a fixed point of $F_{t}^{n}$ in $\Lambda_{n}\left(t_{n}\right)$. We reduce the value of the parameter from $t=t_{n}$ to $t=s$, where $s$ is the value at which the last tangency of $W^{s}\left(p_{n}(s), F_{s}^{n}\right)$ and $W^{u}\left(p_{n}(s), F_{s}^{n}\right)$ occurs. When $t=s$ there still are points for which $W^{s}\left(p_{n}(s), F_{s}^{n}\right)$ and $W^{u}\left(p_{n}(s), F_{s}^{n}\right)$ have transverse intersections.

We now reduce the parameter value by an arbitrarily small amount from $t=s$ to $t=t_{n}^{* *}$. Let $\Lambda_{n}^{\prime}\left(t_{n}^{* *}\right)$ be the hyperbolic set in $B_{n}$ for the map $F_{t}^{n}$ when $t=t_{n}^{* *}$. Note that the Cantor sets of stable and unstable manifolds for $\Lambda_{n}^{\prime}\left(t_{n}^{* *}\right)$ overlap near the bottom of $B_{n}$. We can now measure the thickness of these Cantor sets. When the parameter $t$ is reduced to $t=t_{n}^{* *}$, we have that $\tau^{s}\left(\Lambda_{n}^{\prime}\left(t_{n}^{* *}\right)\right)>T([12$, Chapter VI] or [14]). Also, the stable and unstable manifolds of $\Lambda_{n}^{\prime}\left(t_{n}^{* *}\right)$ and $\Lambda_{1}\left(t_{n}^{* *}\right)$ intersect transversely. This is due to the fact that each iterate of $F_{t}^{n}$ on $W^{u}\left(p_{n}\right)$ extends a local unstable manifold until it crosses $W^{s}\left(P_{t}\right)$ transversely.

We invoke Lemma 8 from [10] to prove that there is a larger hyperbolic set $\Lambda\left(t_{n}^{* *}\right) \supset \Lambda_{1}\left(t_{n}^{* *}\right) \cup \Lambda_{n}^{\prime}\left(t_{n}^{* *}\right)$ for which

$$
\tau^{s}\left(\Lambda\left(t_{n}^{* *}\right)\right) \tau^{u}\left(\Lambda\left(t_{n}^{* *}\right)\right) \geq \tau^{s}\left(\Lambda_{n}^{\prime}\left(t_{n}^{* *}\right)\right) \tau^{u}\left(\Lambda_{1}\left(t_{n}^{* *}\right)\right)>1 .
$$

Let $q_{n}$ be a point on $W^{s}\left(p_{n}\right)$. By Lemma 9 of [10], the Cantor set of unstable manifolds $W^{u}\left(\Lambda\left(t_{n}^{* *}\right)\right)$ can be extended near $q_{n}$ to a foliation $\mathscr{F}^{u}$ with $C^{1}$ tangent vectors. Similarly, $W^{s}\left(\Lambda\left(t_{n}^{* *}\right)\right)$ can be extended near $q_{n}$ to $\mathscr{F}^{s}$. The foliations $\mathscr{F}^{u}$ and $\mathscr{F}^{s}$ will have tangencies of constant finite order and therefore intersect along a $C^{1}$ curve $\gamma$. It is along the curve $\gamma$ for which the local minimum values of $W^{u}$ relative to $W^{s}$ occurs; i.e., $\gamma$ is a curve of potential tangencies. By choosing a small enough curve $\gamma_{\varepsilon}$ that passes through $q_{n}$ and letting $\varepsilon \rightarrow 0$, we have that $\tau\left(W^{s}\left(\Lambda\left(t_{n}^{* *}\right)\right)\right) \tau\left(W^{u}\left(\Lambda\left(t_{n}^{* *}\right)\right)\right)>1$, since the thickness can be measured along any $C^{1}$ curve. Hence, the two Cantor sets intersect and therefore $W^{s}\left(\Lambda\left(t_{n}^{* *}\right)\right)$ has a persistent tangency with $W^{u}\left(\left(t_{n}^{* *}\right)\right)$. This completes the proof of Proposition 1.2, and therefore, the proof of Theorem 1.1.

\section{Proof OF THE MAIN RESULT}

Main Result. Let $\left\{F_{t}\right\}$ be a fixed one parameter family of $C^{\infty}$ diffeomorphisms in two dimensions. Assume that at $t_{0},\left\{F_{t}\right\}$ creates finite order homoclinic 
tangencies for the periodic points $P_{t}$ of period $n$ with

$$
\left|\operatorname{det} D F_{t_{0}}^{n}\left(P_{t_{0}}\right)\right|<1 \quad\left(\left|\operatorname{det} D F_{t_{0}}^{n}\left(P_{t_{0}}\right)>1\right|\right)
$$

and that $F_{t}^{n}$ is $C^{\infty}$ linearizable in a neighborhood of $P_{t}$. Then, for $\varepsilon>0$, there exists a subinterval $\left[t_{1}, t_{2}\right] \subset\left[t_{0}-\varepsilon, t_{0}+\varepsilon\right]$ and a residual subset $J$ in $\left[t_{1}, t_{2}\right]$ such that for $t$ in $J, F_{t}$ has infinitely many sinks (sources).

Proof. If the tangency in the hypothesis of our Main Result is a degenerate homoclinic tangency of constant finite order, then the conclusion of our result follows directly from Theorem 1.1.

Now, assume that the tangency which takes place when $t=t_{0}$ is not a degenerate homoclinic tangency of constant finite order, and suppose that the homoclinic tangency is of order $r$ (where $r=2,4,6, \ldots$ ). For simplicity, we will assume that the tangency occurs at $t=0$.

We introduce new coordinates $(u, v)$ in $U$ such that the $u$-axis lies on the stable manifold of $P_{t}$ and the $v$-axis passes through $q_{0}$. Therefore, a homoclinic tangency takes place at $(0,0)$ when $t=0$. We will express the unstable manifold of $P_{t}$ in a neighborhood of the origin as

$$
\bar{v}_{t}(u)=a_{r} u^{r}+\sum_{i=1}^{r-1} \sum_{j=1}^{\infty}\left(a_{i j} t^{j} u^{i}\right)+t+o\left(t^{2}\right)+O\left(u^{r+1}\right), \quad a_{r} \neq 0,
$$

where at least one of the coefficients $a_{i j}, 1 \leq i \leq r-1$, is not equal to zero. We note that $t \sim \lambda^{-n}$ and that the proof of Theorem 1.1 is uneffected by unfolding terms that involve powers of $t$ greater than or equal to 2 . Hence, we are interested in unfoldings of the type

$$
v_{t}(u)=a_{r} u^{r}+\sum_{i=1}^{r-1} a_{i} t u^{i}+t+o\left(t^{2}\right)+O\left(u^{r+1}\right), \quad a_{r} \neq 0,
$$

where at least one of the coefficients $a_{i}, 1 \leq i \leq r-1$, is not equal to zero. We will now show that as $t$ varies through 0 , new homoclinic tangencies of constant finite order strictly less than $r$ are formed.

Claim. If $s$ is a solution of $v_{t}^{\prime}(u)=0$, then all local extreme values, $v_{t}(s)$, move off the $u$-axis in the same direction for small values of $t$; i.e.,

$$
\left.\frac{\partial v_{t}(s)}{\partial t}\right|_{t=0} \neq 0
$$

To show this, we let $k$ be the integer having the property $a_{k} \neq 0, a_{i}=0$, 
$1 \leq i<k$. Then we have

$$
\begin{aligned}
v_{t}^{\prime}(u) & =r a_{r} u^{r-1}+\sum_{i=1}^{r-1}\left(i a_{i} t u^{i-1}\right)+O\left(u^{r}\right) \\
& =r a_{r} u^{r-1}+\sum_{i=k}^{r-1}\left(i a_{i} t u^{i-1}\right)+O\left(u^{r}\right) \\
& =u^{k-1}\left(r a_{r} u^{r-k}+\sum_{i=k}^{r-1}\left(i a_{i} t u^{i-k}\right)\right)+O\left(u^{r}\right) .
\end{aligned}
$$

The solutions of $v_{t}^{\prime}(u)=0$ are given by $u=0$ or $u \approx s$, where $s$ is a solution of the equation

$$
r a_{r} u^{r-k}+\sum_{i=k}^{r-1}\left(i a_{i} t u^{i-k}\right)=0 .
$$

According to the theory of Newton's polygons [1], $s$ can be expressed in a power series of the form

$$
s=\sum_{p=1}^{\infty} c_{p} t^{p /(r-k)}
$$

where $c_{1} \neq 0$. Evaluating $v_{t}(u)$ when $u=0, s$ yields

$$
v_{t}(0)=t+o\left(t^{2}\right)
$$

and

$$
v_{t}(x)=a_{r}\left(\sum_{p=1}^{\infty} c_{p} t^{p /(r-k)}\right)^{r}+\sum_{i=k}^{r-1} a_{i} t\left(\sum_{p=1}^{\infty} c_{p} t^{p /(r-k)}\right)^{i}+t+o\left(t^{2}\right)+O\left(s^{r+1}\right) .
$$

Differentiating the above equations with respect to $t$ and then evaluating the results at $t=0$ yields

$$
\left.\frac{\partial v_{t}(0)}{\partial t}\right|_{t=0}=1 \neq 0 ;\left.\quad \frac{\partial v_{t}(s)}{\partial t}\right|_{t=0}=1 \neq 0 .
$$

Thus the claim is proven.

Consider an arc of $W^{s}\left(P_{t}, F_{t}\right)$ that is transverse to $W^{u}\left(P_{t}, F_{t}\right)$ in $U$. Let

$$
w(u)=\sum_{i=0}^{r} w_{i} u^{i}+O\left(u^{r+1}\right)
$$

be the Taylor representation for $W^{s}\left(P_{t}, F_{t}\right)$. The $n$th iterate of $w(u)$ is given by

$$
w^{n}(u)=\lambda^{-n} \sum_{i=0}^{r} w_{i} \mu^{i n} u^{i}+O\left(\mu^{n(r+1)} u^{r+1}\right) .
$$

According to the $C^{\infty}$ version of the $\lambda$-lemma [11], iterates of the arc of $W^{s}\left(P_{t}, F_{t}\right)$ converge to the $u$-axis in a $C^{\infty}$ fashion. 
As $t$ varies through 0 , new tangencies between $W^{u}$ and $W^{s}$ are formed. These new tangencies are formed for values of $t$ arbitrarily close to 0 . We will show that these tangencies are of an order strictly less than $r$.

Suppose that the new homoclinic tangency occurs for $u=0$. Then,

$$
v_{t}(u)=a_{r} u^{r}+\sum_{i=k}^{r-1} a_{i} t u^{i}+t+o\left(t^{2}\right)+O\left(u^{r+1}\right)
$$

where $k$ is even. Now we note that the $k$ th derivatives of $v_{t}$ and $w^{n}$ at 0 are given by

$$
\begin{aligned}
v_{t}^{(k)}(0) & =k ! a_{k} t, \\
\left(w^{n}\right)^{(k)}(0) & =\lambda^{-n} \mu^{k n} w_{k} .
\end{aligned}
$$

Since $t \sim \lambda^{-n}$, we have that

$$
v_{t}^{(k)}(0) \approx k ! \lambda^{-n} a_{k}
$$

Hence,

$$
\frac{v_{t}^{(k)}(0)}{\left(w^{n}\right)^{(k)}(0)} \approx \frac{k ! a_{k}}{\mu^{k n} w_{k}} \rightarrow \infty
$$

for large $n$ (small $t$ ). Thus the tangency is of order $k<r$.

Now suppose that the new homoclinic tangency occurs for $u=s \neq 0$. Here we have that

$$
s \approx t^{1 /(r-k)} \approx \lambda^{-n /(r-k)} .
$$

We consider the $(r-1)$ st derivative of $v_{t}$ and $w^{n}$ at $s$;

$$
\begin{gathered}
v_{t}^{(r-1)}(s)=r ! a_{r} \lambda^{-n /(r-k)}+(r-1) ! a_{r-1} \lambda^{-n} \\
\left(w^{n}\right)^{(r-1)}(s)=r ! w_{r} \lambda^{-n} \mu^{r n} \lambda^{-n /(r-k)}+(r-1) ! w_{r-1} \lambda^{-n} \mu^{(r-1) n} .
\end{gathered}
$$

Therefore,

$$
\begin{aligned}
\frac{v_{t}^{(r-1)}(s)}{\left(w^{n}\right)^{(r-1)}(s)} & \approx \frac{r ! a_{r} \lambda^{-n /(r-k)}+(r-1) ! a_{r-1} \lambda^{-n}}{r ! w_{r} \lambda^{-n} \mu^{r n} \lambda^{-n /(r-k)}+(r-1) ! w_{r-1} \lambda^{-n} \mu^{(r-1) n}} \\
& =\frac{r ! a_{r}+(r-1) ! a_{r-1} \lambda^{n(1-r+k) /(r-k)}}{r ! w_{r} \lambda^{-n} \mu^{r n}+(r-1) ! w_{r-1} \lambda^{n(1-r+k) /(r-k)} \mu^{(r-1) n}} \rightarrow \infty
\end{aligned}
$$

for large $n$ (small $t$ ). This implies that the homoclinic tangency at $s$ is of an order strictly less than $r$.

The power series expansions found using the theory of Newton's polygons are convergent in a neighborhood of the origin. Therefore, the new homoclinic tangency when $t$ is near 0 is a degenerate homoclinic tangency of constant finite order. Applying Theorem 1.1 to this new tangency we obtain the desired conclusion. 
Conclusion. Throughout this paper we have focused our attention on the existence of infinitely many coexisting sinks. We have restricted our study to the case where this phenomena results from the formation of homoclinic tangencies in one parameter families of $C^{\infty}$ dissipative diffeomorphisms in the plane. We have shown that they occur whenever the homoclinic tangency is of a finite order. It is unknown at this time whether or not there is another way infinitely many coexisting sinks can result.

\section{REFERENCES}

1. G. A. Bliss, Algebraic functions, Amer. Math. Soc. Colloq. Publ., Vol. 16, Amer. Math. Soc., Providence, R.I., 1933.

2. N. Fenichel, Persistence and smoothness of invariant manifolds for flows, Indiana Univ. Math. J. 21 (1971), 193-226.

3. N. K. Gavrilov and L. P. Silnikov, On three-dimensional dynamical systems close to systems with a structurally unstable homoclinic curve. I, Math. USSR Sb. 17 (1972), 467-485.

4. _. On three-dimensional dynamical systems close to systems with a structurally unstable homoclinic curve. II, Math. USSR Sb. 19 (1973), 139-156.

5. J. Guckenheimer and P. Holmes, Nonlinear oscillations, dynamical systems and bifurcations of vector fields, Appl. Math. Sci., Vol. 42, Springer-Verlag, New York, 1983.

6. M. Hénon, A two-dimensional mapping with a strange attractor, Comm. Math. Phys. $\mathbf{5 0}$ (1976), 69-77.

7. M. W. Hirsch and C. C. Pugh Stable manifolds and hyperbolic sets, Proc. Sympos. Pure Math., vol. 14, Amer. Math. Soc., Providence, R.I., 1970, pp. 133-163.

8. S. E. Newhouse Nondensity of axiom $A(a)$ on $S^{2}$, Proc. Sympos. Pure Math., vol 14, 1970, pp. 191-202.

9. __ Diffeomorphisms with infinitely many sinks, Topology 13 (1974), 9-18.

10. _ The Abundance of wild hyperbolic sets and nonsmooth stable sets for diffeomorphisms, Inst. Hautes Études Sci. Publ. Math. 50 (1979), 101-151.

11. S. E. Newhouse and J. Palis, Cycles and bifurcation theory, Astérisque 31 (1976), 44-140.

12. J. Palis and F. Takens, Homoclinic bifurcations and dynamical systems, Lecture notes, IMPA, 1988.

13. C. Robinson, Bifurcation to infinitely many sinks, Comm. Math. Phys. 90 (1983), 433-459.

14. Corrections to bifurcation to infinitely many sinks, Northwestern University, 1988.

15. S. Smale, Dynamical systems and the topological conjugacy problem for diffeomorphisms, Proc. Internat. Congress Math. (1962), 490-496.

16. S. van Strien, On the bifurcations creating horseshoes, Dynamical Systems and Turbulence, Lecture Notes in Math., vol. 898, Springer-Verlag, Berlin and New York, 1981.

Department of Mathematics, Northwestern University, Evanston, Illinois 60201

Current address: Department of Mathematics, University of Wisconsin-Green Bay, Green Bay, Wisconsin 54311 